\title{
核壳材料 $\mathrm{Co}_{3} \mathrm{O}_{4} @ \mathrm{SiO}_{2}$ 催化环己基过氧化氢分解
}

\author{
陈小梅, 陈 颖, 袁 霞 \\ (湘潭大学 化工学院，湘潭 411105)
}

摘 要: 环己基过氧化氢(CHHP)分解是环己烷无催化氧化工艺制备环己醇和环己酮的重要反应步骤。本研究以 $\mathrm{Co}_{3} \mathrm{O}_{4}$ 纳米颗粒为内核, 十六烷基三甲基溴化铵(CTAB)为模板剂, 正硅酸四乙酯(TEOS)为硅源, 采用模板法制备 了核壳结构材料 $\mathrm{Co}_{3} \mathrm{O}_{4} @ \mathrm{SiO}_{2}$ 。考察了 $\mathrm{SiO}_{2}$ 壳层制备条件: 乙醇和水的比例、 $\mathrm{CTAB}$ 的浓度和 TEOS 的用量对核壳 材料结构的影响。使用不同技术手段表征材料的结构特征, 在 CHHP 分解反应中进行材料的催化性能评价和稳定 性考察。结果表明, 壳层薄且孔隙率高的材料催化性能更好, 并且核壳结构可以减少钴元素的流失, 催化材料在回 收使用过程中出现了不同程度的壳层破碎现象。

关 键 词: 核壳结构; $\mathrm{Co}_{3} \mathrm{O}_{4} @ \mathrm{SiO}_{2} ;$ 环己基过氧化氢；分解

中图分类号: O643 文献标志码: A

\section{Decomposition of Cyclohexyl Hydroperoxide Catalyzed by Core-shell Material $\mathrm{Co}_{3} \mathrm{O}_{4} @ \mathrm{SiO}_{2}$}

\author{
CHEN Xiaomei, CHEN Ying, YUAN Xia
}

(School of Chemical Engineering, Xiangtan University, Xiangtan 411105, China)

\begin{abstract}
Decomposition of cyclohexyl hydroperoxide (CHHP) is an important step in the preparation of cyclohexanol and cyclohexanone by non-catalytic oxidation of cyclohexane. Using $\mathrm{Co}_{3} \mathrm{O}_{4}$ nanoparticles as the core, cetyltrimethylammol/lonium bromide (CTAB) as the template and tetraethyl orthosilicate (TEOS) as the silicon source, the core-shell structure material $\mathrm{Co}_{3} \mathrm{O}_{4} @ \mathrm{SiO}_{2}$ was synthesized by template method. The effects of the preparation conditions for $\mathrm{SiO}_{2}$ shells on the structure of core-shell material were investigated, such as the ratio of ethanol to water, the concentration of CTAB and the amount of TEOS. Textural properties of the materials were characterized by different techniques. Meanwhile, the catalytic performance and stability of the materials were evaluated on the decomposition reaction of CHHP. All results showed that the materials with thin shell and high porosity exhibited a preferable catalytic performance, and the conversion of CHHP was $81.68 \%$ and the selectivity of cyclohexanol and cyclohexanone was $70.60 \%$ and $34.06 \%$, respectively. This core-shell structure could protect the activity of the core $\mathrm{Co}_{3} \mathrm{O}_{4}$ and significantly decrease the loss of cobalt element. However, there existed different degree of the fragmentation of $\mathrm{SiO}_{2}$ shell in the recycling process of $\mathrm{Co}_{3} \mathrm{O}_{4} @ \mathrm{SiO}_{2}$ samples.
\end{abstract}

Key words: core-shell structure; $\mathrm{Co}_{3} \mathrm{O}_{4} @ \mathrm{SiO}_{2}$; cyclohexyl hydroperoxide; decomposition

收稿日期：2021-03-24; 收到修改稿日期：2021-04-23; 网络出版日期：2021-11-15

基金项目: 国家自然科学基金(21776237) National Natural Science Foundation of China (21776237)

作者简介: 陈小梅(1996-), 女, 硕士研究生. E-mail: cxm1077@163.com

CHEN Xiaomei(1996-), female, Master candidate. E-mail: cxm1077@163.com

通信作者: 袁 霞, 教授. E-mail: yuanxia@xtu.edu.cn

YUAN Xia, professor. E-mail: yuanxia@xtu.edu.cn 
环己酮是一种重要的有机化工原料, 主要用于 生产己内酰胺和已二酸, 其需求量逐年增长。目前, 我国环己酮生产以环己烷氧化法和环己烯水合法为 主，其中环己烷氧化法占比更多 ${ }^{[1]}$, 技术成熟。国内 的环己烷氧化法装置多采用 DSM 工艺 ${ }^{[2]}$, 该工艺分 为两步进行: 第一步为环已烷无催化氧化为环己基 过氧化氢(简称 CHHP) 和环已醇、环已酮, 其中环己 烷的转化率约 3.5\%, CHHP 选择性为 $60 \%$ 70\%, CHHP 和醇酮总选择性约 97\%; 第二步为 CHHP 在 碱液和钴盐的作用下, 分解为环己醇和环己酮, 环 己醇脱氢可得环己酮。分解阶段 CHHP 的转化率接 近 $100 \%$, 醇酮选择性约为 $87 \%$, 副反应严重, 而且 废碱液的后处理给环境带来了巨大的压力。因此, 开发一种能在无碱条件下高效分解 CHHP 的催化材 料对于提高环己烷氧化法的竞争力具有重大意义。

CHHP 的分解反应是在变价金属离子催化作用 下的均裂过程 ${ }^{[3-4]}$ 。过渡金属负载型催化材料具备优 异的催化性能, 被广泛使用于 CHHP 分解反应。 Saint-Arroman 等 ${ }^{[5]}$ 制备了一系列 $\mathrm{SiO}_{2}$ 负载型催化剂 $\left.(\equiv \mathrm{SiO})_{3} \mathrm{MOR}, \mathrm{M}=\mathrm{Ti} 、 \mathrm{Zr} 、 \mathrm{Hf}, \mathrm{R}=\mathrm{H}\right)$, 在 $\mathrm{CHHP}$ 分解 反应中负载 $\mathrm{Ti}$ 络合物比负载 $\mathrm{Zr}$ 和 $\mathrm{Hf}$ 络合物活性更 好, 在 $84{ }^{\circ} \mathrm{C}$ 下反应 $6 \mathrm{~h}, \mathrm{CHHP}$ 转化率达到 $85 \%$, 环 己醇和环己酮总选择性达到 $85 \%$ 。然而, 尽管 $\mathrm{Ti}$ 通 过三个共价硅氧钛键固定在 $\mathrm{SiO}_{2}$ 载体上, 仍然会浸 出多达 $30 \%$ 的含量。郭露露等 ${ }^{[6]}$ 采用浸渍法制备了 不同钴负载量的 $\mathrm{Co} / \mathrm{SBA}-15$ 介孔材料, 并将其应用 于 $\mathrm{CHHP}$ 分解反应, $8 \mathrm{wt} \% \mathrm{Co} / \mathrm{SBA}-15$ 在 $70{ }^{\circ} \mathrm{C}$ 下反 应 $90 \mathrm{~min}$, CHHP 分解转化率达到 $98.1 \%$, 环已醇和 环己酮总选择性为 $98.8 \%$ 。钴负载量相对较低时, 钴 的流失严重。当钴负载量增加到 $20 \mathrm{wt} \%$, Co 由骨架 内掺杂状态转变为以 $\mathrm{Co}_{3} \mathrm{O}_{4}$ 形式存在, 钴的流失速 率得到抑制, 一次回收后钴流失约为 $26 \%$ 。如何解 决金属离子流失导致的反应体系结焦堵塞问题是过 渡金属负载型催化材料应用于 CHHP 分解体系需要 重点解决的问题。

核壳结构复合材料广泛应用在生物医药、储能、 气体分离以及催化等领域。 $\mathrm{SiO}_{2}$ 化学性质稳定, 无 毒且具有优异的生物相容性, 易与各种官能团结合 ${ }^{[7]}$, 是壳层的理想选择。Wu 等 ${ }^{[8]}$ 采用改进的 Stöber 法制 备了一种以高活性镍纳米粒子为核, 介孔 $\mathrm{SiO}_{2}$ 为壳 的核壳结构催化材料 $\mathrm{Ni} @ \mathrm{SiO}_{2}$, 该材料在甲烷分解 反应中的稳定性明显优于无硅壳材料和负载型材 料。刘冰等 ${ }^{[9]}$ 采用溶胶-凝胶法, 以 $\mathrm{Fe}_{3} \mathrm{O}_{4}$ 粒子为种 子, 正硅酸四乙酯(TEOS) 为硅源, 通过调节醇水 比、 $\mathrm{NH}_{4} \mathrm{OH}$ 及 TEOS 的浓度, 得到了形貌完整、磁
性可控的核壳结构 $\mathrm{SiO}_{2} / \mathrm{Fe}_{3} \mathrm{O}_{4}$ 复合纳米粒子。

目前还没有关于核壳材料应用于 CHHP 分解的 相关报道, 本工作以 $\mathrm{Co}_{3} \mathrm{O}_{4}$ 为内核, 外层包覆 $\mathrm{SiO}_{2}$ 形成 $\mathrm{Co}_{3} \mathrm{O}_{4} @ \mathrm{SiO}_{2}$ 核壳催化材料应用于 CHHP 分解 反应, 主要探究壳层制备条件对 $\mathrm{Co}_{3} \mathrm{O}_{4} @ \mathrm{SiO}_{2}$ 结构 和性能的影响, 采用多种技术表征催化材料的结构, 并通过 CHHP 分解反应和回收实验评估催化材料的 活性和稳定性。

\section{1 实验方法}

\section{1 实验试剂}

六水合硝酸钴 $\left(\mathrm{Co}\left(\mathrm{NO}_{3}\right)_{2} \cdot 6 \mathrm{H}_{2} \mathrm{O}, \mathrm{AR}\right)$, 正辛醇 (AR), 氨水(质量分数 $25 \%, \mathrm{AR}$ ), 十六烷基三甲基溴 化铵(CTAB, AR), 正硅酸四乙酯(TEOS, AR), 硝酸 铵(AR)均为阿拉丁试剂, 无水乙醇(AR)为湖南汇虹 试剂, 超纯水(自制), 环己烷氧化液 (中石化巴陵分 公司, CHHP 质量分数 $8.39 \%$ )。

\section{2 催化材料的制备}

1) $\mathrm{Co}_{3} \mathrm{O}_{4}$ 内核的制备

将 $5 \mathrm{~g} \mathrm{Co}\left(\mathrm{NO}_{3}\right)_{2} \cdot 6 \mathrm{H}_{2} \mathrm{O}$ 溶解在装有 $500 \mathrm{~mL}$ 正辛 醇的三口烧瓶中, 在油浴锅中加热至 $180{ }^{\circ} \mathrm{C}$, 回流 反应 $2 \mathrm{~h}$, 自然冷却至室温, 离心分离得到沉淀物, 无水乙醇多次洗涤, $80{ }^{\circ} \mathrm{C}$ 鼓风干燥 $3 \mathrm{~h}$, 真空干燥 $10 \mathrm{~h}$ 后得到黑色 $\mathrm{Co}_{3} \mathrm{O}_{4}$ 粉末。

2) $\mathrm{Co}_{3} \mathrm{O}_{4} @ \mathrm{SiO}_{2}$ 复合粒子的制备

配制无水乙醇与超纯水的混合液, 称取 $0.5 \mathrm{~g}$ $\mathrm{Co}_{3} \mathrm{O}_{4}$ 粉末, 分散在装有 $800 \mathrm{~mL}$ 混合液的三口烧瓶 中, 超声处理 $40 \mathrm{~min}$ 形成悬浮液, 在 $30{ }^{\circ} \mathrm{C}$ 磁力摚 拌下, 加入 $30 \mathrm{~mL}$ 氨水, 搅拌 $30 \mathrm{~min}$ 后, 加入 $170 \mathrm{~mL}$ CTAB 的醇水混合液继续摚拌 $1 \mathrm{~h}$, 随后用移液枪吸 取一定体积的 TEOS, 逐滴加入三口烧瓶中。溶液中 无水乙醇的体积比为 $V F_{\mathrm{ETOH}}, \mathrm{CTAB}$ 的浓度为 $C_{\mathrm{CTAB}}$, TEOS 的体积为 $V_{\text {TEOS }}$ $30{ }^{\circ} \mathrm{C}$ 下磁力搅拌 $8 \mathrm{~h}$ 后, 抽 滤分离得到沉淀物, 用超纯水冲洗至洗液呈中性, 将沉淀物干燥后得到 $\mathrm{Co}_{3} \mathrm{O}_{4} @ \mathrm{SiO}_{2}$ 前体。将前体溶 于 $6 \mathrm{~g} / \mathrm{L}$ 的硝酸铵/乙醇混合溶液中, 在 $60{ }^{\circ} \mathrm{C}$ 下回流 搅拌 $2 \mathrm{~h}$, 抽滤分离得到的沉淀物溶于 $300 \mathrm{~mL}$ 乙醇 中, 再在 $60{ }^{\circ} \mathrm{C}$ 下回流搅拌 $1 \mathrm{~h}$, 经抽滤分离得到沉 淀物。重复该操作一次, 将固体 $80{ }^{\circ} \mathrm{C}$ 鼓风干燥 $3 \mathrm{~h}$, 真空干燥 $10 \mathrm{~h}$, 得到的灰褐色粉末记为 $\mathrm{Co}_{3} \mathrm{O}_{4} @ \mathrm{SiO}_{2}$ 。

用上述方法, 制备三个系列的 $\mathrm{Co}_{3} \mathrm{O}_{4} @ \mathrm{SiO}_{2}$, 分 别为: 乙醇的体积比 $V F_{\mathrm{ETOH}}=90 \%, 50 \%, 30 \%$, $\mathrm{CTAB}$ 的浓度为 $14 \mathrm{mmol} / \mathrm{L}$, TEOS 用量为 $1.2 \mathrm{~mL}$; $\mathrm{CTAB}$ 的浓度 $C_{\mathrm{CTAB}}=5,14,25 \mathrm{mmol} / \mathrm{L}$, 乙醇的体积 
比为 $90 \%$, TEOS 用量为 $1.2 \mathrm{~mL}$; 乙醇的体积比为 $90 \%, \mathrm{CTAB}$ 的浓度为 $14 \mathrm{mmol} / \mathrm{L}, \mathrm{TEOS}$ 用量 $V_{\mathrm{TEOS}}=$ $0.8,1.2,2.0 \mathrm{~mL}$ 。

\section{3 催化材料的表征}

采用美国热电集团的 Nicolet-380 型傅里叶红外 光谱仪测定红外光谱(FT-IR), 扫描波数范围为 400 $4000 \mathrm{~cm}^{-1}$ 。采用日本理学公司的 Rigaku D/Max-2550 型 $\mathrm{X}$ 射线衍射仪 (XRD)测定催化材料的物相成分和 晶体结构等, 以 $\mathrm{CuK} \alpha$ 为光源, $\lambda=0.15406 \mathrm{~nm}$, 扫描 速率为 $0.02\left(^{\circ}\right) / \mathrm{min}$, 扫描范围 $2 \theta=5^{\circ} \sim 90^{\circ}$ 。采用 JEOL 的 JEM-2100F 型透射电镜(TEM)观察样品的 精细结构。采用美国 Quantachrome 公司的 NOVA-2200e 物理吸附仪在 $77 \mathrm{~K}$ 液氮温度下测定样 品的物理吸脱附等温线, 样品预处理条件为 $80{ }^{\circ} \mathrm{C}$ 真 空处理 $8 \mathrm{~h}$ 。采用 DFT 模型计算样品的孔径分布曲 线。采用德国 SPECTRO BLUE SOP 全谱直读 ICP 发射光谱仪(ICP)检测催化材料的钴含量。

\subsection{CHHP 分解反应评价}

CHHP 分解反应评价以中石化巴陵分公司的环己 烷氧化液为原料, 各组分的质量分数为: CHHP 8.39\%, 环己醇 $0.79 \%$ ，环己酮 5.49\%，酸 $1.42 \%$ ，酯 $4.98 \%$ 。 在 $50 \mathrm{~mL}$ 三口烧瓶中依次加入磁子、 $25 \mathrm{~g}$ 环己烷氧 化液和适量催化材料, 烧瓶用橡胶塞密封好连接 冷凝管置于 $80{ }^{\circ} \mathrm{C}$ 油浴锅中, 磁力摚拌反应 $1.5 \mathrm{~h}$ 。 将反应液自然冷却, 取上层清液进行产物含量分 析。将离心分离的催化材料用无水乙醇洗涤 3 次, 在 $60{ }^{\circ} \mathrm{C}$ 下鼓风干燥 $3 \mathrm{~h}, 80{ }^{\circ} \mathrm{C}$ 真空干燥 $8 \mathrm{~h}$, 完成催 化材料的回收。

使用碘量滴定法分析反应液中 CHHP 的浓度; 采用福立 9790 II 型气相色谱内标法定量分析环己 醇和环己酮的含量, 采用 DB-1701-1 中极性色谱柱, 内标物为氯苯; 采用化学滴定法测定酸和酯值。

\section{2 结果与讨论}

\section{1 红外光谱(FT-IR)分析}

$\mathrm{Co}_{3} \mathrm{O}_{4} @ \mathrm{SiO}_{2}$ 的红外光谱如图 1 所示, 从图中可 以看出, 样品中均存在位于 1631 与 $3444 \mathrm{~cm}^{-1}$ 处的 吸收峰, 归属于 $\mathrm{SiO}_{2}$ 表面羟基和物理吸附水的 $\mathrm{O}-\mathrm{H}$ 键的弯曲与伸缩振动。样品还均出现了归属于 $\mathrm{Co}_{3} \mathrm{O}_{4}$ 的特征峰, 分别为位于 $663 \mathrm{~cm}^{-1}$ 的 $\mathrm{Co}$ ( II )-O 伸缩振动峰, 以及位于 $577 \mathrm{~cm}^{-1}$ 的 $\mathrm{Co}$ (III) $-\mathrm{O}$ 伸缩振 动峰 ${ }^{[10]}$ 。位于 470、793、1078 以及 $957 \mathrm{~cm}^{-1}$ 处的 特征吸收峰, 分别归属于 $\mathrm{Si}-\mathrm{O}-\mathrm{Si}$ 的弯曲、对称和反 对称伸缩振动以及 $\mathrm{SiO}_{2}$ 表面 $\mathrm{Si}-\mathrm{OH}$ 的伸缩振动 ${ }^{[11]}$,
证明样品中存在 $\mathrm{SiO}_{2}$ 。红外谱图中没有出现位于 2854 以及 $2931 \mathrm{~cm}^{-1}$ 处亚甲基的原位偏振吸收峰 ${ }^{[12]}$, 说明模板剂 CTAB 在萃取洗涤步骤中被除去。

\section{$2.2 \mathrm{X}$ 射线衍射分析}

图 2 为 $\mathrm{Co}_{3} \mathrm{O}_{4} @ \mathrm{SiO}_{2}$ 样品的 XRD 图谱, 样品在 $2 \theta=19.0^{\circ} 、 31.2^{\circ} 、 36.8^{\circ} 、 44.8^{\circ} 、 59.3^{\circ} 、 65.2^{\circ}$ 处均出 现了明显的衍射峰, 分别对应于 $\mathrm{Co}_{3} \mathrm{O}_{4}$ 晶体的(111)、 (220)、(311)、(400)、(511)、(440)晶面。 $\mathrm{SiO}_{2}$ 材料属 于非晶态材料, 没有出现衍射峰。XRD 表征结果说 明 $\mathrm{Co}_{3} \mathrm{O}_{4}$ 内核在包覆和萃取洗涤过程中没有被破坏。

\section{3 透射电镜(TEM)分析}

图 3 是通过改变液相体系中乙醇体积比得到的 $\mathrm{Co}_{3} \mathrm{O}_{4} @ \mathrm{SiO}_{2}$ 样品的 TEM 照片。文献[13-15]研究表 明液相体系中适量的水是核心粒子周围形成均匀、 规则有序的壳层结构的前提。从图 3 中可以看到, 不 同乙醇含量制备得到的 $\mathrm{Co}_{3} \mathrm{O}_{4} @ \mathrm{SiO}_{2}$ 样品, 壳层仍 然呈现致密的无序孔状。三个样品中, $\mathrm{Co}_{3} \mathrm{O}_{4} @ \mathrm{SiO}_{2}$ $\left(V F_{\mathrm{ETOH}}=90 \%\right)$ 的壳层更加均匀、规整, 虽然出现了 部分团聚现象, 但形成的是核包覆壳之后再团聚的 核壳粒子的团聚体(图 3(f)), 区别于 $V F_{\mathrm{ETOH}}=30 \%$ 、 $V F_{\mathrm{ETOH}}=50 \%$ 中核团聚了再包覆壳的多核心核壳粒 子(图 3(b,d))。因此，选择 $V F_{\mathrm{ETOH}}=90 \%$ 作为后续制 备的条件。

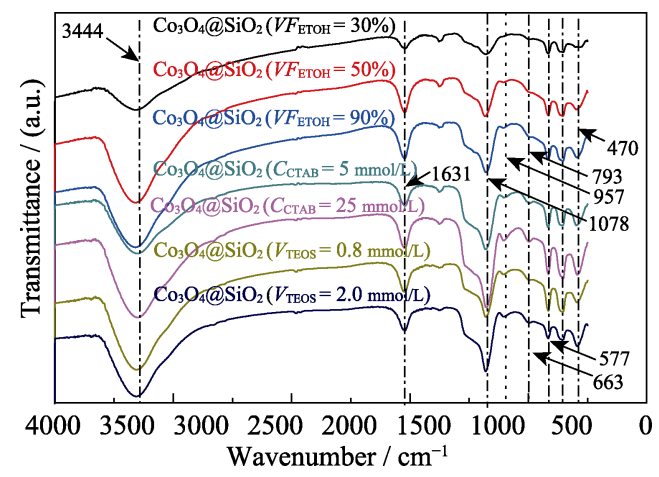

图 1 催化材料的 FT-IR 谱图

Fig. 1 FT-IR spectra of catalytic materials

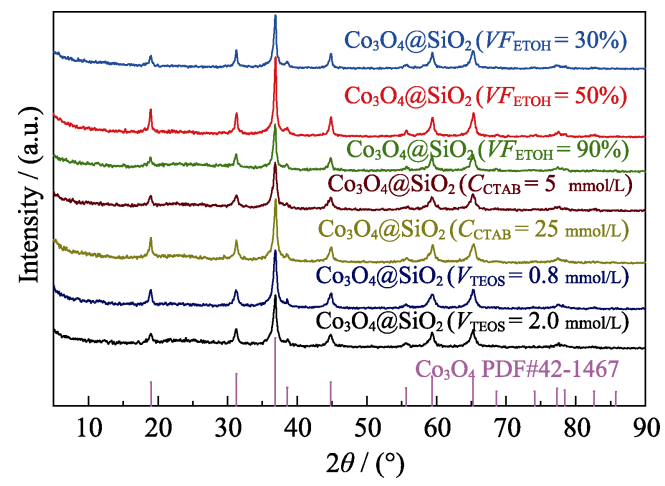

图 2 催化材料的 XRD 图谱

Fig. 2 XRD patterns of catalytic materials 

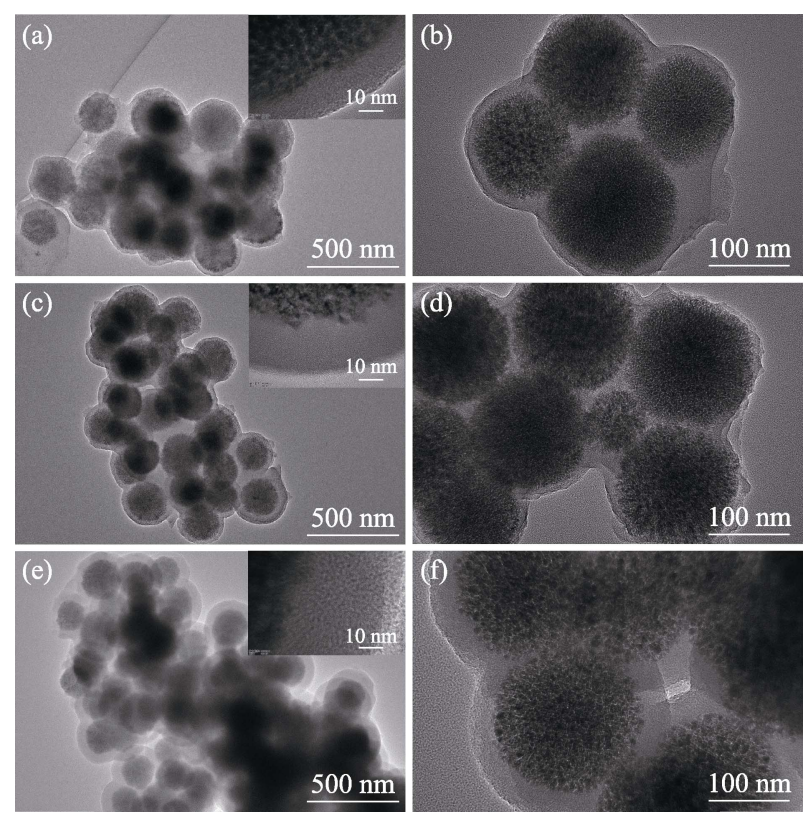

图 3 不同乙醇含量制备的 $\mathrm{Co}_{3} \mathrm{O}_{4} @ \mathrm{SiO}_{2}$ 的 TEM 照片

Fig. 3 TEM images of $\mathrm{Co}_{3} \mathrm{O}_{4} @ \mathrm{SiO}_{2}$ prepared with different ethanol contents

(a, b) $30 \%$; (c, d) $50 \%$; (e, f) $90 \%$

图 4 是通过改变 $\mathrm{CTAB}$ 的加入量制备的 $\mathrm{Co}_{3} \mathrm{O}_{4} @ \mathrm{SiO}_{2}$ 样品的 TEM 照片及其壳层厚度的统计 图, 样品均表现出核壳的包覆结构, 壳层厚度在 $40 \sim 50 \mathrm{~nm}$ 之间的居多, 平均数分布在 47 52 $\mathrm{nm}$ 之 间，这表明 CTAB 作为模板剂，萃取洗涤后从壳层 中洗脱下来，其使用量对壳层厚度基本没有影响。
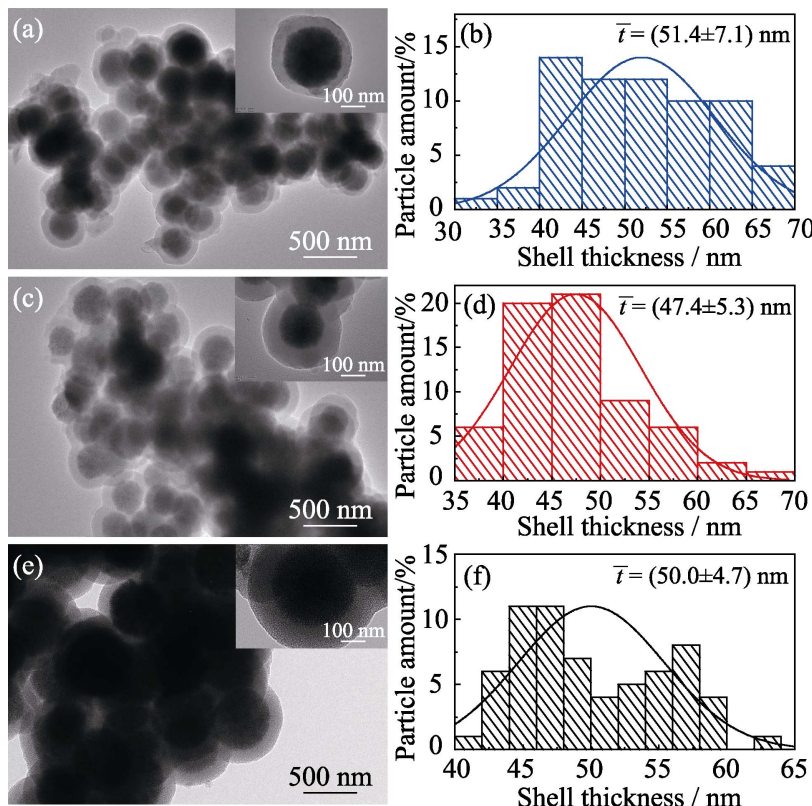

图 4 不同 CTAB 浓度制备的 $\mathrm{Co}_{3} \mathrm{O}_{4} @ \mathrm{SiO}_{2}$ 的 TEM 照片及 其壳层厚度统计图

Fig. 4 TEM images and shell thickness statistics of $\mathrm{Co}_{3} \mathrm{O}_{4} @ \mathrm{SiO}_{2}$ prepared with different concentrations of CTAB (a, b) $5 \mathrm{mmol} / \mathrm{L}$; (c, d) $14 \mathrm{mmol} / \mathrm{L}$; (e, f) $25 \mathrm{mmol} / \mathrm{L}$
图 5 是通过改变 TEOS 的加入量制备的 $\mathrm{Co}_{3} \mathrm{O}_{4} @ \mathrm{SiO}_{2}$ 样品的 TEM 照片及其壳层厚度的统计 图, 样品的壳层厚度仍然主要分布在 40 50 nm 之 间，相比之下，图 5(a)中 $\mathrm{Co}_{3} \mathrm{O}_{4} @ \mathrm{SiO}_{2}\left(V_{\mathrm{TEOS}}=0.8 \mathrm{~mL}\right)$ 的壳层厚度较不均匀, 结合图 5(b)可以发现其壳层 厚度范围为 $25 \sim 85 \mathrm{~nm}$ 。将 TEOS 的用量由 $1.2 \mathrm{~mL}$ 减少至 $0.8 \mathrm{~mL}$ 或增加至 $2.0 \mathrm{~mL}$ 后, 壳层厚度则更多 分布在 25 40 nm 或 50 85 $\mathrm{nm}$ 之间, 这说明 TEOS 用量直接影响 $\mathrm{SiO}_{2}$ 壳层厚度, TEOS 用量越多, 溶液 中水解形成的硅酸根离子多聚体浓度越高, 沉积得 到的 $\mathrm{SiO}_{2}$ 层越厚 ${ }^{[16]}$ 。此外, 壳层厚度的均匀性与 TEOS 的滴加速率有关, 用量减少的情况下更应该 缓慢而均匀地滴加 TEOS。

\section{$2.4 \mathrm{~N}_{2}$ 物理吸附分析}

催化材料的 $\mathrm{N}_{2}$ 物理吸脱附等温线如图 6(a) 所示, 孔径分布如图 6(b)所示, 相应的比表面积和孔的尺 寸数据在表 1 中列出。在图 6(a)中, 根据 IUPAC 分 类, 所有样品都显示出 IV (a)等温线, 表明催化材料 均为介孔材料。

结合图 6(a) 和表 1 可以发现 $\mathrm{Co}_{3} \mathrm{O}_{4} @ \mathrm{SiO}_{2}\left(C_{\mathrm{CTAB}}=\right.$ $25 \mathrm{mmol} / \mathrm{L})$ 和 $\mathrm{Co}_{3} \mathrm{O}_{4} @ \mathrm{SiO}_{2}\left(V_{\mathrm{TEOS}}=2.0 \mathrm{~mL}\right)$ 样品的比 表面积和孔容明显高于其它样品, 这是因为前者制 备过程中添加了更多的 $\mathrm{CTAB}$, 故壳层中有更多来 自 CTAB 架构的孔，而后者制备过程中添加了更多 的 TEOS, 导致催化材料中 $\mathrm{SiO}_{2}$ 的含量更高, 因此二
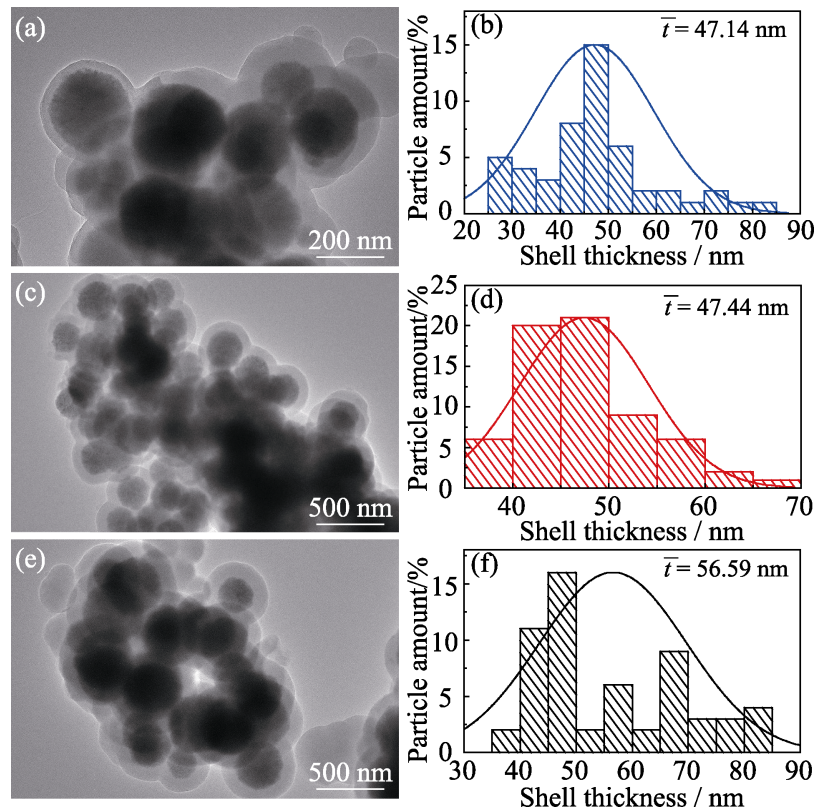

图 5 不同 TEOS 用量制备的 $\mathrm{Co}_{3} \mathrm{O}_{4} @ \mathrm{SiO}_{2}$ 的 TEM 照片及其 壳层厚度统计图

Fig. 5 TEM images and shell thickness statistics of $\mathrm{Co}_{3} \mathrm{O}_{4} @ \mathrm{SiO}_{2}$ prepared with different TEOS dosages (a, b) $0.8 \mathrm{~mL}$; (c, d) $1.2 \mathrm{~mL}$; (e, f) $2.0 \mathrm{~mL}$ 

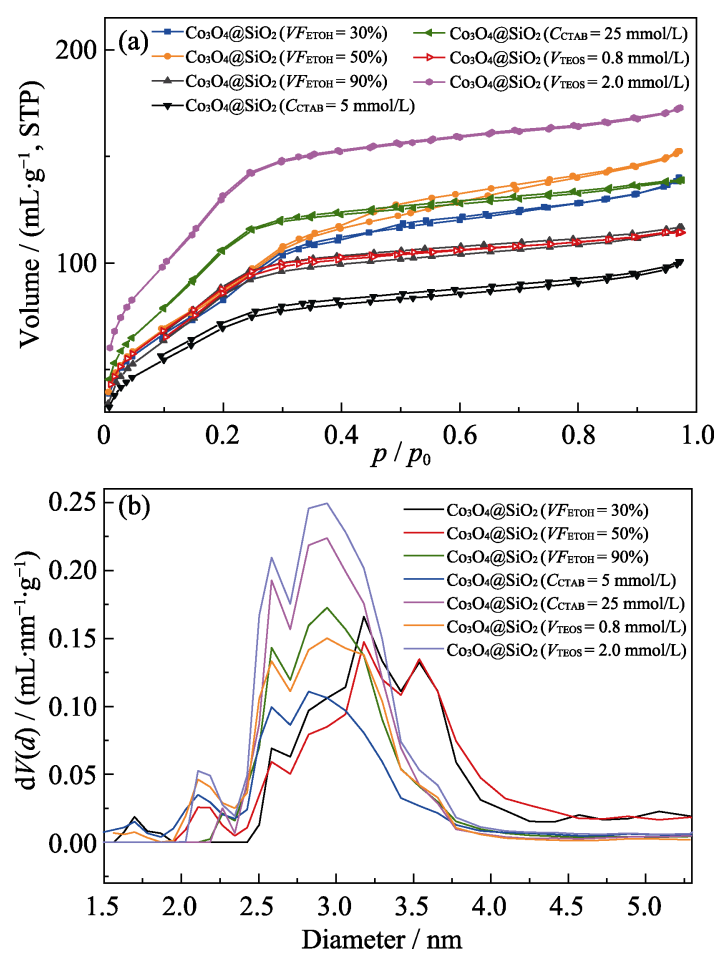

图 6 催化材料的 $\mathrm{N}_{2}$ 物理吸脱附等温线(a)和孔径分布图(b) Fig. $6 \mathrm{~N}_{2}$ physical adsorption and desorption isotherms (a) and pore size distribution (b) of $\mathrm{Co}_{3} \mathrm{O}_{4} @ \mathrm{SiO}_{2}$ Colorful figures are available on website

者的比表面积和孔容更大。与 $\mathrm{Co}_{3} \mathrm{O}_{4} @ \mathrm{SiO}_{2}\left(V F_{\mathrm{ETOH}}=\right.$ $90 \%)$ 相比, $\mathrm{Co}_{3} \mathrm{O}_{4} @ \mathrm{SiO}_{2}\left(C_{\mathrm{CTAB}}=5 \mathrm{mmol} / \mathrm{L}\right)$ 表现出更 小的比表面积和孔容, 因为在制备过程中添加的 $\mathrm{CTAB}$ 越少, 可使壳层中孔隙率越小。而 $\mathrm{Co}_{3} \mathrm{O}_{4} @ \mathrm{SiO}_{2}$ $\left(V_{\mathrm{TEOS}}=0.8 \mathrm{~mL}\right)$ 减少了 TEOS 的用量, 样品的比表面 积和孔容没有减小, 这是因为该样品制备过程中, 表面活性剂与硅源的摩尔比为 3.84, 大于 $\mathrm{Co}_{3} \mathrm{O}_{4} @ \mathrm{SiO}_{2}\left(V F_{\mathrm{ETOH}}=90 \%\right)$ 的 2.56, 其壳层中的孔 隙率略大，同时壳层厚度均匀性较差，部分样品壳 层较厚, 比表面积和孔容并没有变小。从图 6(b)可以 看到, 样品的孔径均集中在 $3 \mathrm{~nm}$ 附近, 其中 $\mathrm{Co}_{3} \mathrm{O}_{4} @ \mathrm{SiO}_{2}\left(V F_{\mathrm{ETOH}}=30 \%\right)$ 和 $\mathrm{Co}_{3} \mathrm{O}_{4} @ \mathrm{SiO}_{2}\left(V F_{\mathrm{ETOH}}=\right.$ $50 \%$ )样品的孔径略大于其它样品, 这可能因为两个 样品在制备过程中溶剂中水的含量更大, 对壳层
$\mathrm{SiO}_{2}$ 有一定蚀刻作用 ${ }^{[17]}$ 。

\section{5 电感偶合等离子体光谱分析}

表 2 是催化材料中 $\mathrm{Co}$ 含量的 ICP 表征结果, 从 条目 1 3 可知, TEOS 用量相同的样品, 钴元素含量 相近; 条目 4 5 表明, TEOS 用量少的样品钴元素含 量相对较高, 说明 TEOS 的用量直接影响 $\mathrm{SiO}_{2}$ 壳层 厚度, 进而影响催化材料中钴的含量, CTAB 的用量 不影响催化材料中的钴含量。

\subsection{CHHP 分解反应评价}

将不同条件下制备的催化材料分别用于 CHHP 的分解反应并进行稳定性考察实验, 按照前期实验 的最佳催化材料用量 $(2 \mathrm{mg} \mathrm{Co} /$ 氧化液)和表 2 结果 折算得到每个催化材料样品的用量。在相同反应条 件下, 新鲜催化材料的反应评价结果如表 3 所示, 其中酯的选择性出现负值, 这是因为在 CHHP 分解 反应中部分酯水解成了环己醇和酸, 导致环己醇和 酸的量增加, 这也造成了醇酮总选择性存在超百的 现象。内核 $\mathrm{Co}_{3} \mathrm{O}_{4}$ 的初始活性最高, 但其反应后容 易团聚，回收一次使用 CHHP 分解转化率下降 $5 \%$ 以上, 而且反应后会粘在反应器壁上难以回收。核 壳材料中 $\mathrm{SiO}_{2}$ 壳层可以保护 $\mathrm{Co}_{3} \mathrm{O}_{4}$ 的活性, 反应后 可以通过简单的方法分离回收使用。 $\mathrm{Co}_{3} \mathrm{O}_{4}$ 和 $\mathrm{Co}_{3} \mathrm{O}_{4} @ \mathrm{SiO}_{2}\left(V_{\mathrm{TEOS}}=0.8 \mathrm{~mL}\right)$ 反应液中的 $\mathrm{Co}$ 元素含 量分别为 $5.66 \mu \mathrm{g} / \mathrm{g}$ 和 $4.12 \mu \mathrm{g} / \mathrm{g}$, 说明 $\mathrm{SiO}_{2}$ 的包覆可 以减缓钴元素流失。

核壳材料中 $\mathrm{Co}_{3} \mathrm{O}_{4} @ \mathrm{SiO}_{2}\left(V_{\mathrm{TEOS}}=0.8 \mathrm{~mL}\right)$ 转化 率最高, 该样品平均壳层厚度最薄, 且比表面积和 孔容较大, 反应物的扩散阻力较小, 较容易接近活 性内核。活性最低的是 $\mathrm{Co}_{3} \mathrm{O}_{4} @ \mathrm{SiO}_{2}\left(V F_{\mathrm{ETOH}}=30 \%\right)$, 该样品壳层制备时溶液中水的比例较大，导致 $\mathrm{Co}_{3} \mathrm{O}_{4}$ 内核团聚之后再包覆 $\mathrm{SiO}_{2}$ 壳层, 这使得活性 中心没有完全分散，与反应物的接触不够充分。 $\mathrm{Co}_{3} \mathrm{O}_{4} @ \mathrm{SiO}_{2}\left(V F_{\mathrm{ETOH}}=50 \%\right)$ 制备时溶液中水的比 例也较 $\mathrm{Co}_{3} \mathrm{O}_{4} @ \mathrm{SiO}_{2}\left(V F_{\mathrm{ETOH}}=90 \%\right)$ 大, 但是初始活 性却较高, 这是因为其壳层孔径较大。

\section{表 1 催化材料的物理结构特性}

Table 1 Physical structural properties of catalytic materials

\begin{tabular}{|c|c|c|c|c|}
\hline Sample & $n_{\mathrm{CTAB}} / n_{\mathrm{TEOS}}$ & $S_{\mathrm{BET}} /\left(\mathrm{m}^{2} \cdot \mathrm{g}^{-1}\right)$ & $D_{\text {pore }} / \mathrm{nm}$ & $V_{\text {pore }} /\left(\mathrm{cm}^{3} \cdot \mathrm{g}^{-1}\right)$ \\
\hline $\mathrm{Co}_{3} \mathrm{O}_{4} @ \mathrm{SiO}_{2}\left(V F_{\mathrm{ETOH}}=30 \%\right)$ & 2.56 & 332 & 3.18 & 0.22 \\
\hline $\mathrm{Co}_{3} \mathrm{O}_{4} @ \mathrm{SiO}_{2}\left(V F_{\mathrm{ETOH}}=50 \%\right)$ & 2.56 & 334 & 3.18 & 0.24 \\
\hline $\mathrm{Co}_{3} \mathrm{O}_{4} @ \mathrm{SiO}_{2}\left(V F_{\mathrm{ETOH}}=90 \%\right)$ & 2.56 & 319 & 2.94 & 0.18 \\
\hline $\mathrm{Co}_{3} \mathrm{O}_{4} @ \mathrm{SiO}_{2}\left(C_{\mathrm{CTAB}}=5 \mathrm{mmol} / \mathrm{L}\right)$ & 0.93 & 254 & 2.82 & 0.16 \\
\hline $\mathrm{Co}_{3} \mathrm{O}_{4} @ \mathrm{SiO}_{2}\left(C_{\mathrm{CTAB}}=25 \mathrm{mmol} / \mathrm{L}\right)$ & 4.67 & 396 & 2.94 & 0.21 \\
\hline $\mathrm{Co}_{3} \mathrm{O}_{4} @ \mathrm{SiO}_{2}\left(V_{\mathrm{TEOS}}=0.8 \mathrm{~mL}\right)$ & 3.84 & 327 & 2.94 & 0.18 \\
\hline $\mathrm{Co}_{3} \mathrm{O}_{4} @ \mathrm{SiO}_{2}\left(V_{\mathrm{TEOS}}=2.0 \mathrm{~mL}\right)$ & 1.54 & 486 & 2.94 & 0.27 \\
\hline
\end{tabular}


表 2 催化材料的 Co 元素含量

Table 2 Co content of catalytic materials

\begin{tabular}{clc}
\hline Entry & \multicolumn{1}{c}{ Sample } & Co content $/ \%$ \\
\hline 1 & $\mathrm{Co}_{3} \mathrm{O}_{4} @ \mathrm{SiO}_{2}\left(V F_{\mathrm{ETOH}}=90 \%\right)$ & 39.9 \\
2 & $\mathrm{Co}_{3} \mathrm{O}_{4} @ \mathrm{SiO}_{2}\left(C_{\mathrm{CTAB}}=5 \mathrm{mmol} / \mathrm{L}\right)$ & 40.1 \\
3 & $\mathrm{Co}_{3} \mathrm{O}_{4} @ \mathrm{SiO}_{2}\left(C_{\mathrm{CTAB}}=25 \mathrm{mmol} / \mathrm{L}\right)$ & 39.3 \\
4 & $\mathrm{Co}_{3} \mathrm{O}_{4} @ \mathrm{SiO}_{2}\left(V_{\mathrm{TEOS}}=0.8 \mathrm{~mL}\right)$ & 47.8 \\
5 & $\mathrm{Co}_{3} \mathrm{O}_{4} @ \mathrm{SiO}_{2}\left(V_{\mathrm{TEOS}}=2.0 \mathrm{~mL}\right)$ & 30.6 \\
\hline
\end{tabular}

$\mathrm{Co}_{3} \mathrm{O}_{4} @ \mathrm{SiO}_{2}\left(V_{\mathrm{TEOS}}=2.0 \mathrm{~mL}\right)$ 和 $\mathrm{Co}_{3} \mathrm{O}_{4} @ \mathrm{SiO}_{2}$ $\left(C_{\mathrm{CTAB}}=5 \mathrm{mmol} / \mathrm{L}\right)$ 的壳层制备条件 $n_{\mathrm{CTAB}} / n_{\mathrm{TEOS}}$ 较小, 即模板剂比例较低。前者的 $\mathrm{SiO}_{2}$ 壳层最厚, 但仍表 现出较高的初始活性, 这是因为在首次使用过程中 壳层出现了明显破碎的现象, 如图 7 所示。这导致 $\mathrm{Co}_{3} \mathrm{O}_{4}$ 内核暴露出来与反应物直接接触, 从而获得 了较高的 CHHP 转化率。而后者初始活性仅为 $55.62 \%$, 这与其较小的比表面积和孔容相一致。上 述结果说明模板剂比例较低不利于得到催化性能较 好的 $\mathrm{Co}_{3} \mathrm{O}_{4} @ \mathrm{SiO}_{2}$ 。

图 8 是催化材料样品的稳定性考察数据。 $\mathrm{Co}_{3} \mathrm{O}_{4} @ \mathrm{SiO}_{2}\left(V_{\mathrm{TEOS}}=0.8 \mathrm{~mL}\right)$ 的壳层最薄, 其初始活 性最好, 随着反应次数的增多, 对 CHHP 分解的转 化率逐渐下降, 这是由于在重复使用过程中, Co 元 素发生流失。 $\mathrm{Co}_{3} \mathrm{O}_{4} @ \mathrm{SiO}_{2}\left(V F_{\mathrm{ETOH}}=30 \%\right)$ 、 $\mathrm{Co}_{3} \mathrm{O}_{4} @ \mathrm{SiO}_{2}\left(V F_{\mathrm{ETOH}}=50 \%\right)$ 和 $\mathrm{Co}_{3} \mathrm{O}_{4} @ \mathrm{SiO}_{2}\left(V F_{\mathrm{ETOH}}=\right.$ 90\%)随着反应次数的增多, 活性呈现出先上升后下 降的变化趋势, 这可能是由于它们的壳层相对较厚, 反应过程中传质阻力较大, 导致催化材料存在诱导 期, 所以前几次反应活性逐渐上升。文献 ${ }^{[18]}$ 中 $\mathrm{Au} / \mathrm{SiO}_{2}$ 在 $\mathrm{CO}$ 氧化反应中也存在同样的现象。后续 反应活性下降也与钴元素的流失有关。 $\mathrm{Co}_{3} \mathrm{O}_{4} @ \mathrm{SiO}_{2}$ $\left(V F_{\mathrm{ETOH}}=90 \%\right) 5$ 次反应前后的 $\mathrm{Co}$ 含量由 $39.9 \%$ 下
降至 33.6\%。 $\mathrm{Co}_{3} \mathrm{O}_{4} @ \mathrm{SiO}_{2}\left(C_{\mathrm{CTAB}}=25 \mathrm{mmol} / \mathrm{L}\right)$ 重复 使用 5 次, CHHP 分解转化率一直呈上升趋势, 5 次 反应后样品的 TEM 表征结果如图 9 所示, 从图中可 以看到部分核壳颗粒形貌完好, 大部分核壳粒子壳 层厚度变薄, 一部分壳层破碎, 从而导致 $\mathrm{Co}_{3} \mathrm{O}_{4}$ 内 核更容易与反应物接触, 这可能是导致转化率一直 上升的原因。

\section{3 结论}

本研究制备了核壳结构的催化材料 $\mathrm{Co}_{3} \mathrm{O}_{4} @ \mathrm{SiO}_{2}$, 探讨壳层制备条件对催化材料结构的影响, 并在 CHHP 分解反应中进行催化性能评价和稳定性考察, 得出如下结论:

1) $\mathrm{Co}_{3} \mathrm{O}_{4} @ \mathrm{SiO}_{2}$ 的制备过程中, 乙醇水溶液中 水含量大于 $10 \%$ 以上, 可以增大壳层无序中孔的孔 径, 但容易造成 $\mathrm{Co}_{3} \mathrm{O}_{4}$ 内核的团聚以及壳层结构的 不稳定;

2) $\mathrm{SiO}_{2}$ 壳层中的孔隙率随 CTAB 与 TEOS 摩尔 比的增大而增大; 壳层的厚度不受 $\mathrm{CTAB}$ 用量影响, 而与 TEOS 用量有关, 缓慢滴加 TEOS 有利于形成厚 度均匀的壳层;

3)壳层厚度更薄和孔隙率更高的催化材料样品 $\mathrm{Co}_{3} \mathrm{O}_{4} @ \mathrm{SiO}_{2}\left(V_{\mathrm{TEOS}}=0.8 \mathrm{~mL}\right)$ 在 $\mathrm{CHHP}$ 分解反应中性 能最好, 转化率为 $81.68 \%$, 环已醇和环己酮的选择 性分别为 $70.60 \%$ 和 $34.06 \%$;

4) $\mathrm{SiO}_{2}$ 壳层包覆可以有效提高 $\mathrm{Co}_{3} \mathrm{O}_{4}$ 的回收率, 减缓钴元素的流失, 但是 $\mathrm{Co}_{3} \mathrm{O}_{4} @ \mathrm{SiO}_{2}$ 在回收使用过 程中出现了不同程度的破碎现象, $\mathrm{SiO}_{2}$ 壳层对 $\mathrm{CHHP}$ 分解反应过程的耐受度还需进一步加强。

表 3 催化材料在 CHHP 分解反应中的催化性能

Table 3 Catalytic performance of catalytic materials in CHHP decomposition reaction

\begin{tabular}{|c|c|c|c|c|c|c|}
\hline \multirow{2}{*}{ Catalytic material } & \multirow{2}{*}{ Con. $/ \%$} & \multicolumn{5}{|c|}{ Selectivity $/ \%$} \\
\hline & & $\mathrm{A}$ & $\mathrm{K}$ & Acid & Ester & $\mathrm{A}+\mathrm{K}$ \\
\hline Blank & 11.58 & 162.27 & 36.16 & 12.56 & 14.40 & 198.43 \\
\hline $\mathrm{Co}_{3} \mathrm{O}_{4}$ & 86.83 & 69.15 & 38.08 & 6.73 & -15.63 & 107.23 \\
\hline $\mathrm{Co}_{3} \mathrm{O}_{4} @ \mathrm{SiO}_{2}\left(V F_{\mathrm{ETOH}}=30 \%\right)$ & 41.54 & 66.93 & 37.70 & 8.14 & -26.54 & 104.62 \\
\hline $\mathrm{Co}_{3} \mathrm{O}_{4} @ \mathrm{SiO}_{2}\left(V F_{\mathrm{ETOH}}=50 \%\right)$ & 60.44 & 67.73 & 32.87 & 7.33 & -18.46 & 100.60 \\
\hline $\mathrm{Co}_{3} \mathrm{O}_{4} @ \mathrm{SiO}_{2}\left(V F_{\mathrm{ETOH}}=90 \%\right)$ & 52.62 & 64.58 & 30.20 & 8.54 & -25.15 & 94.79 \\
\hline $\mathrm{Co}_{3} \mathrm{O}_{4} @ \mathrm{SiO}_{2}\left(C_{\mathrm{CTAB}}=5 \mathrm{mmol} / \mathrm{L}\right)$ & 55.62 & 74.72 & 36.68 & 6.59 & -20.69 & 111.40 \\
\hline $\mathrm{Co}_{3} \mathrm{O}_{4} @ \mathrm{SiO}_{2}\left(C_{\mathrm{CTAB}}=25 \mathrm{mmol} / \mathrm{L}\right)$ & 66.68 & 72.18 & 31.01 & 6.83 & -15.76 & 103.19 \\
\hline $\mathrm{Co}_{3} \mathrm{O}_{4} @ \mathrm{SiO}_{2}\left(V_{\mathrm{TEOS}}=0.8 \mathrm{~mL}\right)$ & 81.68 & 70.60 & 34.06 & 7.12 & -15.97 & 104.65 \\
\hline $\mathrm{Co}_{3} \mathrm{O}_{4} @ \mathrm{SiO}_{2}\left(V_{\mathrm{TEOS}}=2.0 \mathrm{~mL}\right)$ & 73.55 & 64.75 & 27.90 & 6.28 & -18.74 & 92.64 \\
\hline
\end{tabular}

Reactions conditions: $2 \mathrm{mg}$ of Co per gram of cyclohexane oxide (containing $8.39 \% \mathrm{CHHP}), T=80{ }^{\circ} \mathrm{C}$, time $=90 \mathrm{~min}$; A: cyclohexanol; $\mathrm{K}$ : cyclohexanone 

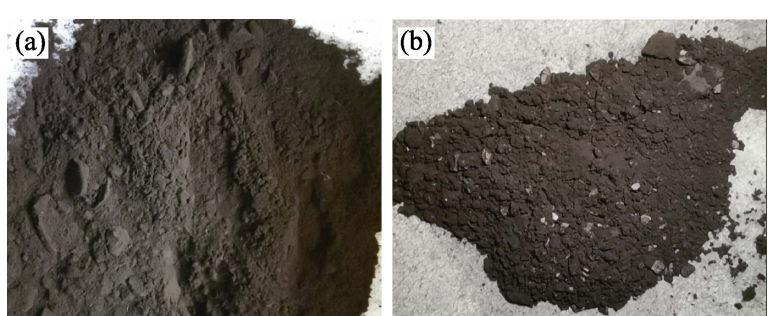

图 $7 \mathrm{Co}_{3} \mathrm{O}_{4} @ \mathrm{SiO}_{2}\left(V_{\mathrm{TEOS}}=2.0 \mathrm{~mL}\right)$ 反应前后的外观对比图

Fig. 7 Appearance comparison of $\mathrm{Co}_{3} \mathrm{O}_{4} @ \mathrm{SiO}_{2}\left(V_{\text {TEOS }}=2.0 \mathrm{~mL}\right)$ before and after the reaction

(a) Fresh; (b) $1^{\text {st }}$ cycle

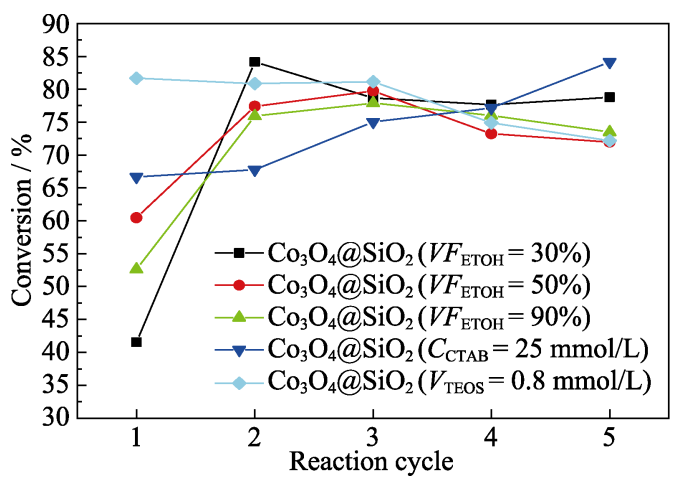

图 8 不同催化材料的新鲜及回收样品的反应评价结果

Fig. 8 Reaction evaluation results of fresh and recovered samples catalytic materials

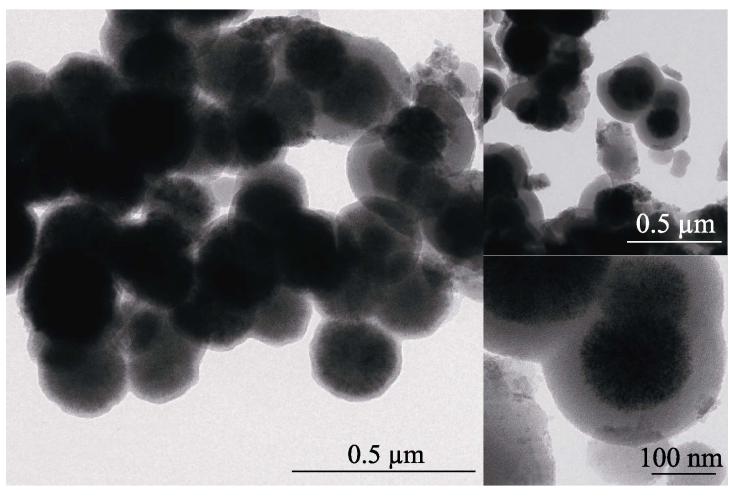

图 9 反应 5 次后回收 $\mathrm{Co}_{3} \mathrm{O}_{4} @ \mathrm{SiO}_{2}\left(C_{\mathrm{CTAB}}=25 \mathrm{mmol} / \mathrm{L}\right)$ 的 TEM 照片

Fig. 9 TEM images of $\mathrm{Co}_{3} \mathrm{O}_{4} @ \mathrm{SiO}_{2}\left(C_{\mathrm{CTAB}}=25 \mathrm{mmol} / \mathrm{L}\right)$ recovered after 5 cycles

\section{参考文献:}

[1] 黄瑞丽. 国内环己酮生产现状及消费分析. 合成纤维工业, 2020, 43(4): 63-67.

[2] 宋星星, 李永祥, 吴巍. 环已基过氧化氢分解工艺技术现状和 发展. 化工进展, 2004, 23(3): 322-325.

[3] IMANAKA N, MASUI T, JYOKO K. Selective liquid phase oxidation of cyclohexane over $\mathrm{Pt} / \mathrm{CeO}_{2}-\mathrm{ZrO}_{2}-\mathrm{SnO}_{2} / \mathrm{SiO}_{2}$ catalysts with molecular oxygen. Journal of Advanced Ceramics, 2015, 4(2):
111-117.

[4] KIRILlOVA M V, KOZLOV Y N, SHUL'PINA L S, et al. Remarkably fast oxidation of alkanes by hydrogen peroxide catalyzed by a tetracopper(II) triethanolaminate complex: promoting effects of acid co-catalysts and water, kinetic and mechanistic features. Journal of Catalysis, 2009, 268(1): 26-38.

[5] SAINT-ARROMAN R P, BASSET J M, LEFEBVRE F, et al. Well-defined group IV supported catalysts: an efficient way to increase activities in the deperoxidation of cyclohexyl hydroperoxide with environmentally systems. Applied Catalysis A: General, 2005, 290(1/2): 181-190.

[6] GUO LULU, LI LIXIA, HE PENGCHENG, et al. Mesoporous material Co/SBA-15 as catalyst for the decomposition of cyclohexyl hydroperoxide. Journal of Inorganic Materials, 2017, 32(5): 543549.

[7] OBAIDULLAHA M, BAHADURB N M, FURUSAWAA T, et al. Microwave assisted rapid synthesis of $\mathrm{Fe}_{2} \mathrm{O}_{3} @ \mathrm{SiO}_{2}$ core-shell nanocomposite for the persistence of magnetic property at high temperature. Colloids and Surfaces A: Physicochemical and Engineering Aspects, 2019, 572: 138-146.

[8] WU S L, YANG R X, WEY M Y. Catalytic methane decomposition to hydrogen over a surface-protected core-shell $\mathrm{Ni@SiO} \mathrm{SiO}_{2}$ catalyst. Chemical Engineering \& Technology, 2018, 41(7): 1448-1456.

[9] LIU BING, WANG DEPING, HUANG WENHAI, et al. Preparation of core-shell $\mathrm{SiO}_{2} / \mathrm{Fe}_{3} \mathrm{O}_{4}$ nanocomposite particles via Sol-Gel approach. Journal of Inorganic Materials, 2008, 23(1): 33-38.

[10] PEJOVA B, ISAHI A, NAJDOSKI M, et al. Fabrication and characterization of nanocrystalline cobalt oxide thin films. Materials Research Bulletin, 2000, 36(2001): 161-170.

[11] ZHAO L, SHI S, LIU M, et al. Hydrophobic modification of microenvironment of highly dispersed $\mathrm{Co}_{3} \mathrm{O}_{4}$ nanoparticles for the catalytic selective oxidation of ethylbenzene. ChemCatChem, 2019, 12(3): 903-910.

[12] NEIVANDT D J, GEE M L, HAIR M L. Polarized infrared attenuated total reflection for the in situ determination of the orientation of surfactant adsorbed at the solid/solution interface. $J$. Phys. Chem. B, 1998, 102(26): 5107-5114.

[13] KIM J H, YOON S B, KIM J Y, et al. Synthesis of monodisperse silica spheres with solid core and mesoporous shell: morphological control of mesopores. Colloids and Surfaces A: Physicochemical and Engineering Aspects, 2008, 313-314: 77-81.

[14] YOON S B, KIM J Y, KIM J H, et al. Synthesis of monodisperse spherical silica particles with solid core and mesoporous shell: mesopore channels perpendicular to the surface. Journal of Materials Chemistry, 2007, 17(18): 1758-1761.

[15] YIN L, TIAN Q, BOYJOO Y, et al. Synthesis of colloidal mesoporous silica spheres with large through-holes on the shell. Langmuir, 2020, 36(25): 6984-6993.

[16] DING H L, ZHANG Y X, WANG S, et al. $\mathrm{Fe}_{3} \mathrm{O}_{4} @ \mathrm{SiO}_{2}$ core/shell nanoparticles: the silica coating regulations with a single core for different core sizes and shell thicknesses. Chemistry of Materials, 2012, 24(23): 4572-4580.

[17] TENG Z, SU X, ZHENG Y, et al. Mesoporous silica hollow spheres with ordered radial mesochannels by a spontaneous self-transformation approach. Chemistry of Materials, 2012, 25(1): 98-105.

[18] ZHU H, MA Z, CLARK J C, et al. Low-temperature CO oxidation on $\mathrm{Au} /$ fumed $\mathrm{SiO}_{2}$-based catalysts prepared from $\mathrm{Au}(\mathrm{en})_{2} \mathrm{Cl}_{3}$ precursor. Applied Catalysis A: General, 2007, 326(1): 89-99. 